\title{
A Case of Rudimentary Horn Pregnancy Diagnosed After Failed Attempts at Pregnancy Termination
}

\author{
Fatemeh Aliasghari ${ }^{1}$ and Raheleh Rezaei ${ }^{1,{ }^{*}}$ \\ ${ }^{1}$ Department of Obstetrics and Gynecology, Ali-Ebn-Abitaleb Hospital, Zahedan University of Medical Sciences, Zahedan, Iran \\ "Corresponding author: Department of Obstetrics and Gynecology, Ali-Ebn-Abitaleb Hospital, Zahedan University of Medical Sciences, Zahedan, Iran. Email: \\ rezaei_98@yahoo.com
}

Received 2017 December 27; Accepted 2018 March 04.

\begin{abstract}
We present a case of rudimentary horn pregnancy in the 18th gestational week with fetal demise and treatment failure. After failure in pregnancy termination, the patient was admitted to a tertiary care hospital. Following laparatomy, a definitive diagnosis was confirmed. Laparotomy was performed to remove the rudimentary horn and ipsilateral fallopian tube. Overall, physicians should be cautious about the risk of uterine disorders in the event of several failures in terminating pregnancy.
\end{abstract}

Keywords: Müllerian Anomalies, Pregnancy Termination, Rudimentary Horn Pregnancy, Unicornuate Uterus

\section{Introduction}

Uterine abnormalities are usually asymptomatic and remain undiagnosed in early years of life.

According to statistics, one in every 4000 women is diagnosed with a unicornuate uterus in the general population (1). Although a unicornuate uterus can be an isolated finding, it is commonly accompanied by a noncommunicating rudimentary horn (2).

Pregnancy in women with a noncommunicating uterine horn seems to be a result of transperitoneal sperm migration from the contralateral tube, which fertilizes the ova in the fallopian tube of the rudimentary horn (1). It is very important to diagnose a rudimentary horn pregnancy as early as possible, as it can result in a fatal bleeding in case of uterine rupture (3).

Herein, we report a diagnosis of rudimentary horn pregnancy after failed attempts to terminate the pregnancy.

\section{Case Presentation}

An 18-year-old girl (G2P2D1; NVD) was admitted to our center at 18 weeks of gestation. The patient had experienced menarche at the age of 15 and reported normal menstruation without dysmenorrhea. She had a history of preterm labor in which the neonate died (at six months of gestation). Intrauterine fetal demise was diagnosed in the patient and labor was induced with misoprostol. Induction was unsuccessful after 48 hours and she was referred to our hospital with failed termination of pregnancy.

Her vital signs and general condition were normal upon admission. A single cervix was detected on the speculum examination, and abdominal ultrasounds showed an empty uterus (bicornuate uterus) and a nonviable fetus at the gestational age of 18 weeks. The patient was suspected of a rudimentary horn pregnancy. She consented to a laparotomy after failed attempts at termination of pregnancy, a rudimentary horn pregnancy was found intraoperatively on the right side (Figure 1).

After making a vertical incision on the anterior surface of the horn, the cavity was evacuated.

Cavity exploration showed no outflow of pathways and the fallopian tube and right rudimentary horn were finally removed. After an uneventful postoperative period, she was discharged from the hospital two days after surgery.

\section{Discussion}

Partial development of a Müllerian duct and its incomplete fusion with the contralateral side result in a rudimentary horn and a unicornuate uterus. A unicornuate uterus belongs to class II of the American Fertility Society categorization, subdivided into four subgroups: Class IIA, communication between the rudimentary horn cavity and the uterus; class IIB, lack of communication between the cavity of the rudimentary horn and the uterus; class IIC, no cavity 


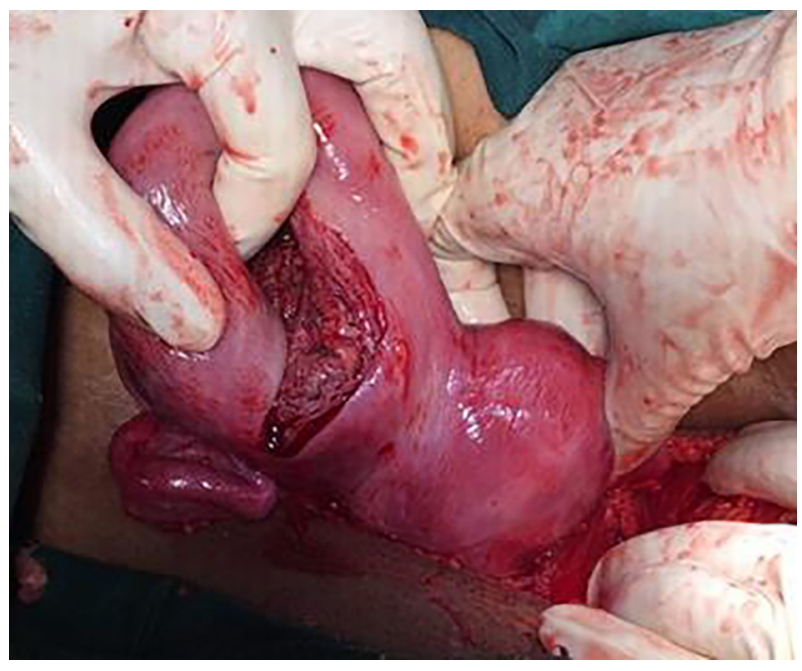

Figure 1. Rudimentary horn pregnancy on the right side

in the rudimentary; and class IID, no horn (4). The present case was categorized as class IID.

Approximately $2.4 \%$ - $13 \%$ of all Müllerian anomalies are attributed to a unicornuate uterus (5). In $74 \%$ of unicornuate uteri, rudimentary horns can be detected (6). The rudimentary horn is non-communicating in $83 \%$ of patients (7). According to the literature, rudimentary horns tend to be on the right side (57-80\%), as observed in the present case (7). This Müllerian anomaly is associated with significant obstetric risks, including first-and secondtrimester miscarriage, mal presentation, fetal growth restriction, fetal demise, prematurely ruptured membranes, and preterm delivery. If diagnosed in a non-pregnant woman, prophylactic excision of the horn with a cavity is recommended. Importantly, $40 \%$ of affected women will have renal anomalies, and evaluation of these cases is necessary (1).

\subsection{Conclusion}

Maternal and fetal health may be extremely compromised as a consequence of pregnancy in a rudimentary uterine horn. We can improve the assessment of this uncommon condition through careful ultrasonography in the first trimester. MRI is recognized as a helpful diagnostic test, which can improve clinician consultations prior to surgery. The possibility of this type of pregnancy should be considered in women with unsuccessful attempts after pregnancy.

\section{References}

1. Cunningham L, Bloom H, Hauth R. Williams obstetrics. 24th Ed. New York: Mc Graw Hill; 2014. p. 40-1.

2. Siwatch S, Mehra R, Pandher DK, Huria A. Rudimentary horn pregnancy: A 10-year experience and review of literature. Arch Gynecol Obstet. 2013;287(4):687-95. doi: 10.1007/s00404-012-2625-7. [PubMed: 23183713].

3. Fritz M, Speroff L. Clinical gynecologic endocrinology and infertility. 8th Ed. New York: Wolters Kluwer; 2011.

4. The American Fertility Society. The American Fertility Society classifications of adnexal adhesions, distal tubal occlusion, tubal occlusion secondary to tubal ligation, tubal pregnancies, mullerian anomalies and intrauterine adhesions. Fertil Steril. 1988;49(6):944-55. doi: 10.1016/S0015-0282(16)59942-7. [PubMed: 3371491].

5. Simon C, Martinez L, Pardo F, Tortajada M, Pellicer A. Mullerian defects in women with normal reproductive outcome. Fertil Steril. 1991;56(6):1192-3. doi: 10.1016/S0015-0282(16)54741-4. [PubMed: 1743344].

6. Nahum GG. Uterine anomalies. How common are they, and what is their distribution among subtypes? J Reprod Med. 1998;43(10):877-87. [PubMed: 9800671].

7. Heinonen PK. Unicornuate uterus and rudimentary horn. Fertil Steril.1997;68(2):224-30.doi:10.1016/S0015-0282(97)81506-3. [PubMed: 9240247]. 